\title{
Pseudomyogenic Hemangioendothelioma
}

\author{
Shin-ichi Ansai ${ }^{1}$, Maya Morimoto ${ }^{2}$ and Satoshi Akaishi ${ }^{2}$
}

\begin{abstract}
${ }^{1}$ Division of Dermatology and Dermatopathology, Nippon Medical School Musashi Kosugi Hospital, Kanagawa, Japan
${ }^{2}$ Division of Plastic and Reconstructive Surgery, Nippon Medical School Musashi Kosugi Hospital, Kanagawa, Japan
\end{abstract}

Pseudomyogenic hemangioendothelioma (PMHE) is a new entity. It is an intermediate soft tissue tumor clinically and/or histopathologically mimicking some other high-grade malignant tumors and some inflammatory diseases. We report a case of PMHE on the left plantar surface of a 28-year-old woman. Histopathological examination of the resected specimen revealed spindle and epithelioid cells with plump and atypical nuclei proliferated in the dermis and subcutaneous fat tissue with marked fibroplasia. Both spindle and epithelioid cells had abundant eosinophilic cytoplasm. Neoplastic cells were diffusely positive for AE1/AE3, CK7, vimentin, CD31, FLI-1, ERG, and INI-1. From those findings, we made the diagnosis of PMHE. We describe the main points of differentiation between PMHE and diseases that have similar clinical and/or histopathological findings, including cellular dermatofibroma, spindle cell squamous cell carcinoma, epithelioid sarcoma, epithelioid hemangioendothelioma, epithelioid angiosarcoma, nodular or proliferative fasciitis, and granulomatous fibrosing granulation tissue due to a ruptured epidermal cyst. (J Nippon Med Sch 2019; 86: 126-130)

Key words: pseudomyogenic hemangioendothelima, epithelioid sarcoma, keratin, CD31

\section{Introduction}

Pseudomyogenic hemangioendothelioma (PMHE) is a relatively new entity, first reported in 1992 by Mirra et al. as a fibroma-like variant of epithelioid sarcoma ${ }^{1}$. In 2003, Billings et al. reported seven other cases that were similar to those of Mirra et al. and proposed to designate this tumor epithelioid sarcoma-like hemangioendothelioma ${ }^{2}$. Hornick and Fletcher presented 29 other similar cases, which they named pseudomyogenic (fibroma-like) variants of epithelioid sarcoma ${ }^{3}$. Subsequently, they published a series of 50 cases confirming its vascular differentiation and indolent behavior and designated it $\mathrm{PMHE}^{4}$. Although this neoplasm may be extremely rare, it frequently arises on the dermis and/or subcutis of extremities of young adults. Furthermore, it is classified into rarely metastasizing, vascular neoplasms of intermediate malignant potential in the current World Health Organization classification of tumors of soft tissue and bone ${ }^{5}$. However, dermatologists, dermatologic surgeons, and dermatopathologists may have little knowledge of this tumor. We report a case of this neoplasm arising on the plantar surface of a young woman.

\section{Case Report}

A 28-year-old woman visited the outpatient clinic of Plastic and Reconstructive Surgery of Nippon Medical School Musashi Kosugi Hospital complaining of a tender nodule on her left plantar surface for several months. She had no notable prior medical problems or trauma. Physical examination at that time revealed a hard elastic intradermal nodule $5 \mathrm{~mm}$ in diameter. The surface of the lesion was slightly erythematous. Surgeons suspected a ruptured epidermal cyst and the lesion was resected with a minimal margin.

Histopathological examination of the resected specimen revealed spindle and epithelioid cells with plump and atypical nuclei proliferated throughout the dermis and subcutaneous fat tissue (Fig. 1a) with marked fibroplasia (Fig. 1b). The lesion was ill-defined and myxoid stromal changes were observed in some areas (Fig. 1c). Neoplastic cells were arranged in sheets or fascicles. Both spindle and epithelioid cells had abundant eosinophilic

Correspondence to Shin-ichi Ansai, MD, PhD, Division of Dermatology, Nippon Medical School Musashi Kosugi Hospital, 1396 Kosugi-cho, Nakahara-ku, Kawasaki, Kanagawa 211-8533, Japan

E-mail: shin8113@nms.ac.jp

https://doi.org/10.1272/jnms.JNMS.2019_86-209

Journal Website (http://www2.nms.ac.jp/jnms/) 

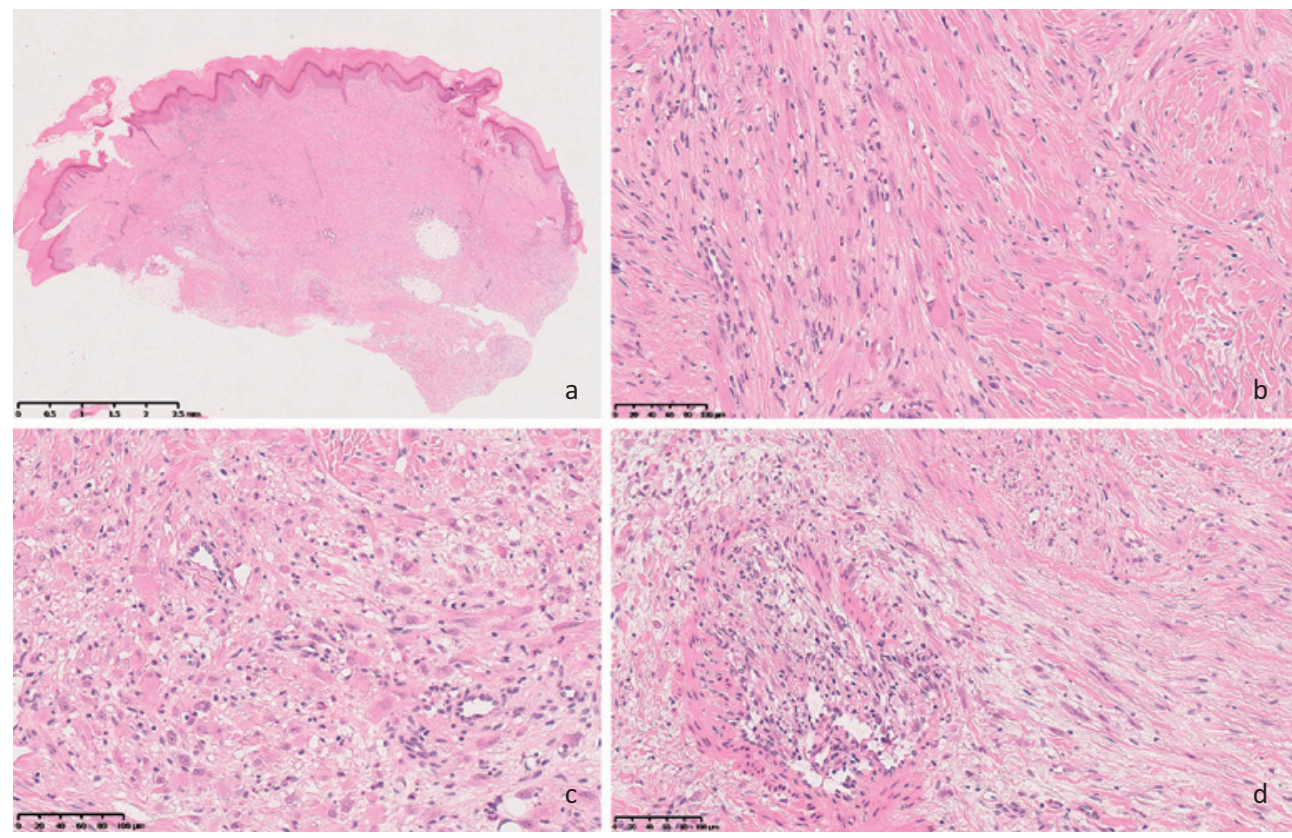

Fig. 1

Histopathological examination of the resected specimen revealed that the ill-defined lesion existed in the dermis and subcutaneous fat tissue with marked fibroplasia (a). The lesion is composed of spindle (b) and epithelioid cells (c) with plump and atypical nuclei. Myxoid changes of stroma are observed in some areas (a, c, d). Neoplastic cells are arranged in sheets or fascicles (b, c). Both spindle and epithelioid cells have abundant eosinophilic cytoplasm (b, c). In some areas, vascular structures that are entrapped within the tumor show invasion of their lumina by neoplastic cells (d). (HE staining)

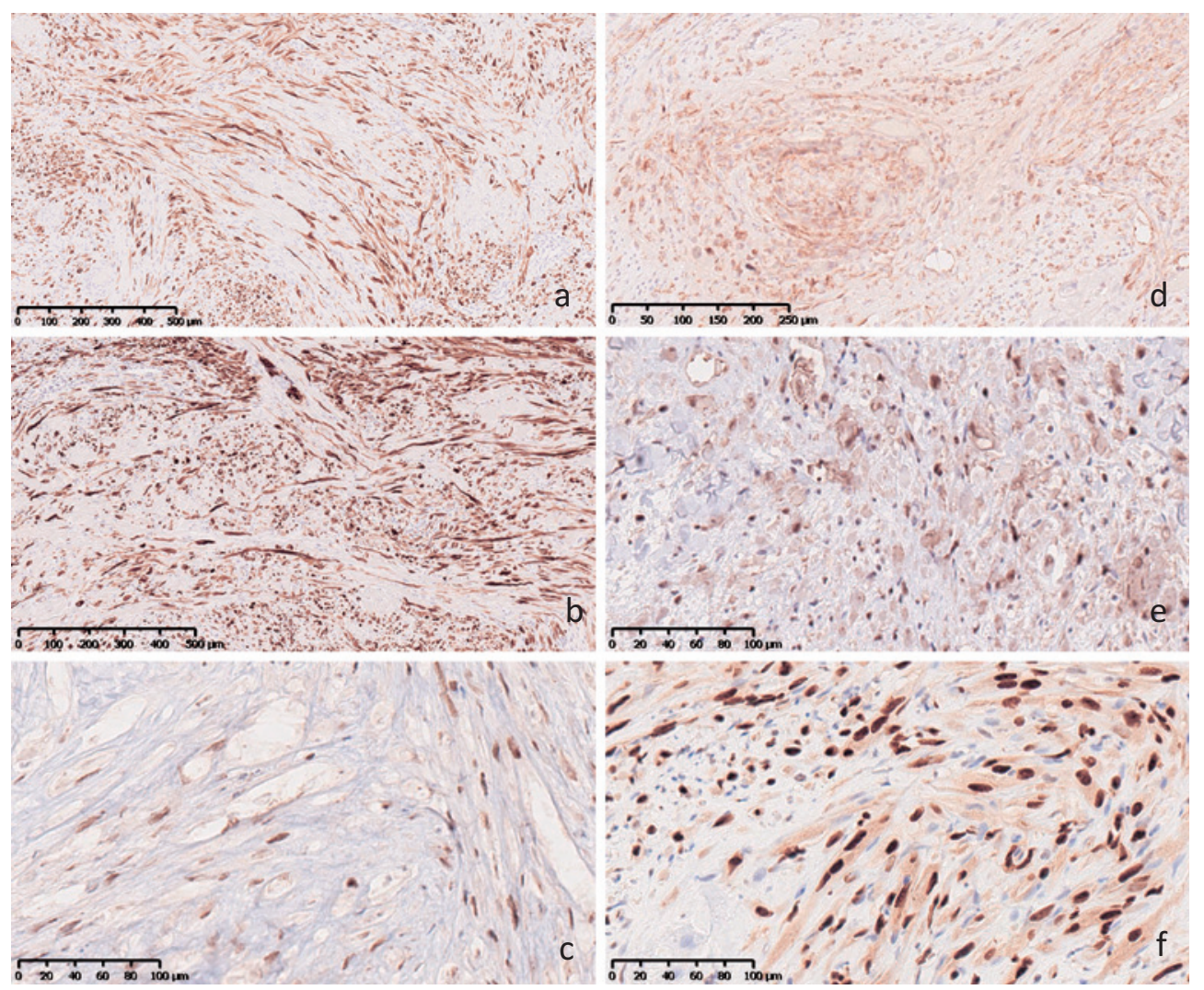

Fig. 2

Neoplastic cells are positive for AE1/AE3 (a), CK7 (b), INI-1 (c), CD31 (d), FLI-1 (e), and ERG (f). (Immunoperoxidase stain for a: AE1/AE3, b: CK7, C: INI-1, d: CD31, e: FLI-1, and f: ERG) 
cytoplasm (Fig. 1b, c). Mitotic figures were rarely observed. In some areas, vascular structures that were entrapped within the tumor showed invasion of their lumina by neoplastic cells (Fig. 1d). Small numbers of inflammatory cells, including neutrophils, were observed throughout the lesion.

Neoplastic cells of this case were diffusely positive for cytokeratin (CK) (AE1/AE3) (Fig. 2a), CK 7 (Fig. 2b), INI-1 (Fig. 2c), CD31 (Fig. 2d), FLI-1 (Fig. 2e), ERG (Fig. 2f) and vimentin, and partially for $\alpha$-smooth muscle actin and CD68 (KP-1). They were negative for CD34, S-100 protein, desmin, synaptophysin, chromogranin $\mathrm{A}$, and glial fibrillary acidic protein. Almost all the tumor cells were positive for p53, whereas the Ki-67 labeling index was approximately 20 to $30 \%$. Based on these findings, we made a diagnosis of PMHE. No recurrence has been observed six months after the resection.

\section{Discussion}

The clinical characteristics of PMHE have been reported by several authors ${ }^{4-8}$. The tumor occurs more frequently in males than in females, with the peak incidence in young adults. It often affects the lower extremities (about $60 \%$ ), and the upper extremities and trunk are less commonly involved. Half of the patients with this tumor complain of pain and half are asymptomatic. In approximately two-thirds of the patients, PMHE occurs multifocally, often affecting multiple tissue planes. The lesions of most patients are in the dermis and/or subcutaneous tissue. About half of the patients with PMHE have intramuscular lesions and $20 \%$ of the patients have lytic bone lesions.

The histopathological characteristics of PMHE have also been summarized ${ }^{4-8}$. In most instances, the lesion shows an infiltrative growth pattern that extends into the adjacent tissue. Sometimes, the tumors contain focally myxoid stroma. Neoplastic cells often look like rhabdomyoblasts, because they exhibit characteristic features and have oval or round, abundant eosinophilic cytoplasm. They also have vesicular nuclei and variable nucleoli. Cytologic atypia are rarely observed, and mitotic figures are infrequently seen. Occasionally, there is an inflammatory infiltrate of neutrophils throughout the lesion.

PMHE exhibits unique immunohistochemical findings $^{4-8}$. The neoplastic cells of PMHE are usually positive for endothelial markers such as CD31, FLI-1, and ERG, whereas they are negative for CD34. They also coexpress vimentin and keratins such as AE1/AE3, CK7, CAM5.2, and MNF116. INI-1 and epithelial membrane antigen are often positive in tumor cells, though they are negative for S-100 protein and desmin. Sometimes, they may be partially positive for $\alpha$-smooth muscle actin. The immunohistochemical findings of our case were consistent with the above. Some cytogenetic investigations have been reported so far. The chromosomal translocation $\mathrm{t}(7 ; 19)(\mathrm{q} 22$; q13) leading to SERPINE1-FOSB gene fusion had been identified in some cases ${ }^{8-10}$.

Differential diagnosis of PMHE includes several benign, intermediate, and malignant diseases as well as inflammatory disorders. The major differential diagnoses include cellular dermatofibroma, spindle cell squamous cell carcinoma, epithelioid sarcoma (ES), epithelioid hemangioendothelioma (EHE), epithelioid angiosarcoma (EAS), nodular or proliferative fasciitis, other myogenic tumors, and various other smooth muscle and myofibroblastic tumors. Granulomatous fibrosing granulation tissue due to a ruptured epidermal cyst should also be included. We indicate the main points of differentiation in the Table and below.

The rhabdomyoblast-like cells are not observed in cellular dermatofibroma and its neoplastic cells are negative for cytokeratin and CD31, $1^{4,811}$. Spindle cell squamous cell carcinoma usually occurs in sun-damaged skin of the elderly, typically shows striking nuclear atypia, and its neoplastic cells do not express endothelial markers. Neoplastic cells of myofibroblastic tumors are negative for keratin and extremely rarely express endothelial mark$\mathrm{ers}^{4,8}$.

Initially, Mirra et $\mathrm{al}^{1}$. thought that PMHE was a variant of ES. ES resembles the demographic, clinical, and pathologic features of PMHE in that it often affects young adults with multifocal disease and involves skin and soft tissue of the extremities. ES is composed of keratinpositive epithelioid cells, which are, in a subset of cases, positive for ERG and FLI1. Although INI1 is lost in most ESs, it is positive in all PMHEs. The lack of immunoreactivity for CD34 in PMHE may also be valuable for differentiation of those two neoplasms.

EHE is an angiocentric tumor comprising cords of epithelioid cells, some with intracytoplasmic vacuoles usually embedded in a myxohyaline stroma ${ }^{12}$. EHE is usually positive for CD34, though PMHE is negative for it.

In some instances, neoplastic cells of EAS also strongly express keratin and endothelial markers like those of PMHE. However, EAS is an aggressive tumor with vasoformative architecture, a sheet-like growth pattern, striking nuclear atypia, frequent mitotic figures, and irregular 


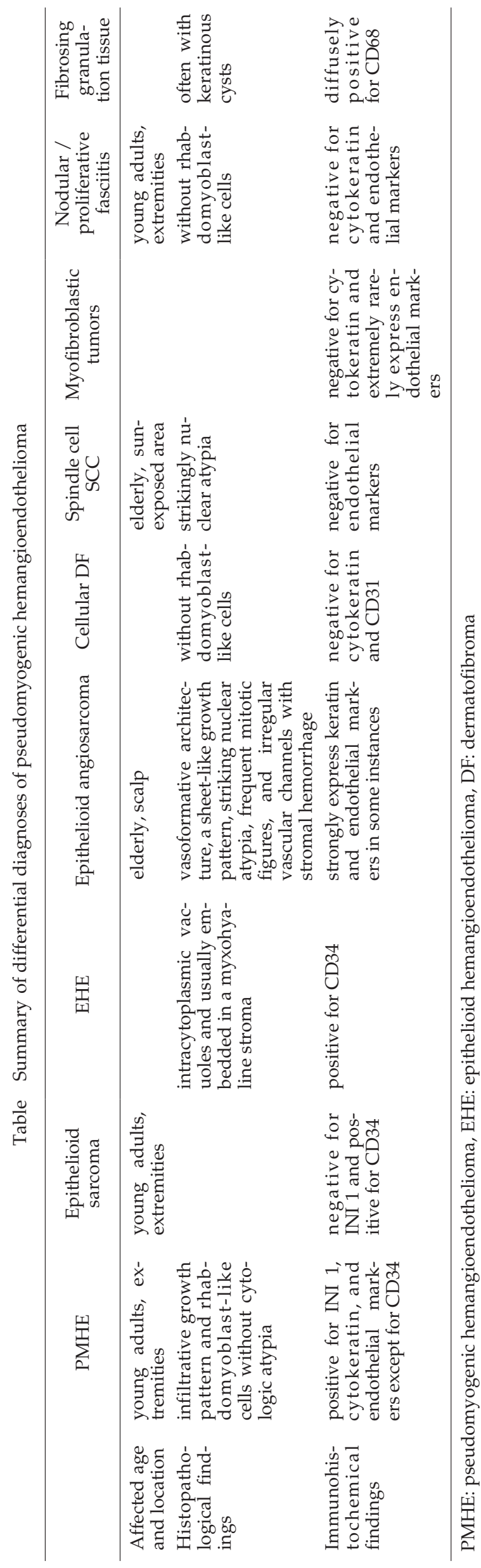

vascular channels with stromal hemorrhage, unlike $\mathrm{PMHE}^{13}$.

Nodular/proliferative fasciitis generally lacks the rhabdomyoblast-like cells, diffuse keratin expression, and FLI1 and/or CD31 positivity. PMHE lacks keratinous cysts and its epithelioid cells do not diffusely express CD68, whereas those findings should be observed in granulomatous fibrosing granulation tissue due to ruptured epidermal cysts.

PMHE is an extremely rare neoplasm; however, dermatologists, dermatologic surgeons, and dermatopathologists should be aware of it.

Acknowledgments: The authors wish to thank Dr. Keisuke Goto and Dr. Takaya Fukumoto for their valuable advice in diagnosis of this case, and Dr. Keiko Miura for her help in immunohistochemical work.

Conflict of Interest: none decleared

\section{References}

1. Mirra JM, Kessler S, Bhuta S, Eckardt J: The fibroma-like variant of epithelioid sarcoma: a fibrohistiocytic/myoid cell lesion often confused with benign and malignant spindle cell tumors. Cancer 1992; 69: 1382-1395.

2. Billings SD, Folpe AL, Weiss SW: Epithelioid sarcoma-like hemangioendothelioma. Am J Surg Pathol 2003; 27: 48-57.

3. Hornick JL, Fletcher CD: Pseudomyogenic ("fibromalike") variant of epithelioid sarcoma: a distinctive tumor type with a propensity for multifocality in a single limb but surprisingly indolent behavior. Mod Pathol 2008; 21: 13.

4. Hornick JL, Fletcher CDM: Pseudomyogenic hemangioendothelioma: a distinctive, often multicentric tumor with indolent behavior. Am J Surg Pathol 2011; 35: 190-201.

5. Hornick JL, Fletcher CDM, Mertens F: Pseudomyogenic hemangioendothelioma. In World Health Organization classification of tumors of soft tissue and bone. Pathology and genetics of tumors of soft tissue and bone (Fletcher CDM, Bridge JA, Hogendoorn PCW, Mertens F, eds), 2013; pp 153-154, IARC Press, Lyon.

6. Hung YP, Fletcher CDM, Hornick JL: FOSB is a useful diagnostic marker for Pseudomyogenic hemangioendothelioma. Am J Surg Pathol 2017; 41: 596-606.

7. Pradhan D, Schoedel K, McGough RL, Ranganathan S, Rao UNM: Pseudomyogenic hemangioendothelioma of the skin, bone and soft tissue-a clinicopathological, immunohistochemical, and fluorescence in situ hybridization study. Hum Pathol 2018; 71: 126-134.

8. Requena L, Santonja C, Martinez-Amo JL, Saus C, Kutzner H: Cutaneous epithelioid sarcoma-like (pseudomyogenic) hemangioendothelioma. A little known low grade cutaneous vascular neoplasm. JAMA Dermatol 2013; 149: 459-465.

9. Trombetta D, Magnusson L, von Steyern FV, Hornick JL, Fletcher CDM, Mertens F: Translocation t(7;19)(q22;q13)-a recurrent chromosome aberration in pseudomyogenic hemangioendothelioma? Cancer Genet 2011; 204: 211-215.

10. Walther C, Tayebwa J, Lilljebjorn H, Magnusson L, 
Nilsson J, von Steyem FV, Ora I, Domanski HA, Fioretos $\mathrm{T}$, Nord $\mathrm{KH}$, Fletcher CDM, Mertens F: A novel SERPINE 1-FOSB fusion gene results in transcriptional upregulation of FOSB in pseudomyogenic haemangioendothelioma. J Pathol 2014; 232: 534-540.

11. Sheng W, Pan Y, Wang J: Pseudomyogenic hemangioendothelioma: report of an additional case with aggressive clinical course. Am J Dermatopathol 2013; 35: 597-600.

12. Mentzel T, Beham A, Calonje E, Katenkamp D, Fletcher C: Epithelioid hemangioendothelioma of skin and soft tissues: clinicopathologic and immunohistochemical study of 30 cases. Am J Surg Pathol 1997; 21: 363-374.

13. Fletcher CD, Beham A, Bekir S, Clarke A, Marley N: Epithelioid angiosarcoma of deep soft tissue: a distinctive tumor readily mistaken for an epithelial neoplasm. Am J Surg Pathol 1991; 15: 915-924.

(Received, August 17, 2018) (Accepted, November 8, 2018) 